\title{
Design and fabrication of solar panel with sun position tracker
}

\author{
Firas B. Ismail1 ${ }^{1}$, Nizar F.O. Al-Muhsen² ${ }^{2}$ Fazreen A. Fuzi ${ }^{1}$, S. Sambathan ${ }^{1}$ and Muhammad N. H. Nawawi ${ }^{1}$ \\ 1 Power Generation Unit, Institute of Power Engineering, Universiti Tenaga Nasional, Jalan IKRAM-UNITEN, 43000 Kajang, Selangor, Malaysia \\ Phone: +60389212020 Ext: 6637 \\ 2 Technical Instructors Training Institute, Middle Technical University, Baghdad, Iraq
}

\begin{abstract}
Timelessly, the sun has been paramount source of energy for life on earth and a large portion of the energy is utilized just for warmth and lighting. Decisively, daylight can be utilized as another form of energy by completely harvesting the light rays from the sun into a reliable sustainable source of energy. The main objective of this work is to develop a new solar panel design with better energy harvesting efficiency with the capability of tracking the position of the sun using real-time tracker. Three solar panels are stacked above one another without overshadowing the below ones. Solar concentrator is also employed to focus the sun irradiance onto panels. To ensure maximum power harvested, solar panel needs to be perpendicular to the sun's array. Therefore, solar panels are rotated using combination of servo motors, Real Time Clock (RTC) and Arduino Mega 2560 to certain angles at certain period. The result is then compared with the conventional solar panel system, and it is found that the new design generates $50 \mathrm{~kW} / \mathrm{h}$ extra energy, which is about $21.24 \%$ greater than the static conventional system. The overall increment of the average output power is about $28.5 \%$ which is for all the working hours over three days' testing time. Besides, it is found that the performance of the proposed system could be influenced by the operating temperature of the PV modules.
\end{abstract}

ARTICLE HISTORY

Revised: $11^{\text {th }}$ May 2020

Accepted: $15^{\text {th }}$ May 2020

\section{KEYWORDS}

Sun Position Tracker; multilevel PV system; rotating PV panels.

\section{INTRODUCTION}

The notion of photovoltaic effect could be traced back to Edmund Becquerel in 1839 [1]. He observed that when direct light strikes one of the electrodes of an electrolyte process, voltage was generated, and he called that the effect photovoltaic. Since then, a new method of generating electricity was invented. Photovoltaic (PV) was first commercially used in 1954 for the purpose of monitoring satellite in space. Recently, the demand for PV panel has increased drastically over the last few decades due to its significant capability to convert solar energy into electricity [2]. Annually, planet Earth receives by up to $16 \times 1018$ units of solar energy [3]. This demonstrates that the sun has been represented as the most promising renewable source of energy for human beings. Unlike fossil fuels, it is an environmentally friendly and very clean energy source, and it does not produce pollutions [4]. However, the unpredictable weather and fixed-tilt angle could potentially reduce the output power of the PV systems. To increase the energy conversion efficiency and reduce the operational cost of any conventional PV module, the penetration of the solar irradiance needs to be increased during the day time $[5,6]$. This could be achieved by using a sun position tracking system provided with an accurate algorithm that should be able to follow the sun path precisely over time [7].

A multilevel PV system is a new approach to improve the conversion efficiency of the PV systems, and it was proposed by Debnath T. et al. [8]. By implementing real time clock (RTC), the multilevel solar panel would rotate by 15 degrees with a total of 12 positions to locate the sun. These three layers of solar panels would not overshadow each another as they were initially mounted in slanted position, with the middle panel turned vertical in the afternoon. This system could harness by $20-23 \%$ greater energy meanwhile it was occupying about $33 \%$ smaller area than the conventional static solar panel system. A PV system with solar tracker integrated with a solar concentrator was also investigated by Kabir M. S. et al. [9]. The main solar concentrator was made of two flat mirrors. In addition, four flat mirrors were also used to further enhance the output power of the PV system. These solar concentrators were designed to collect the incident lights from the sun and focus them on the PV panel. Besides, a Fuzzy Logic Controller was used to move the solar panel to the targeted angle toward the sun. The average output power of the used PV system was increased by 33.50 to $58.32 \%$ when the solar concentrators were used.

Light Dependent Resistor (LDR) was used in sun tracking system by Sharma V. and Tayal V. K. [10]. The LDR was embedded in the same platform as solar panel, thus enabling it to detect highest light intensity. The major outcome of this module was producing $32 \%$ higher output than flat solar panel. The usage of this sun tracker was a feasible technique to harness the optimum energy from the penetrated solar irradiance. In May 2015, a new design of sun tracker was introduced which was based on Arduino microcontroller plus a stepper motor, GPS module, and mirrors to reflect sunlight towards the PV panels. The GPS module was used to ensure the position of the solar panel was directly perpendicular to the sunlight. An Arduino microcontroller is used to interpret the data collected by tracking system, then run the stepper motor aiming for the sun. However, the stepper motor requires a specific power to operate reducing the overall efficiency of the 
system [11]. Meikap S. and Dey S. had developed a low-cost optimum power tracking prototype for solar energy [12]. The prototype was a single axis Arduino based solar tracker and consisted of five main hardware; RTC, Arduino microcontroller, DC geared motor, DC motor driver and PV panel. Initially, the solar panel was tilted into 22.5 degrees North and later was rotated according to RTC to locate the sun. An analog signal from RTC goes to Arduino according to the time, and calculations of zenith and elevation angle were also carried out. The main advantages of this prototype are the tracker could follow the sun accurately and it could be implemented anywhere.

It could be observed from the aforementioned in the literature that several research works were performed targeting a maximum amount of solar energy generation for improving the energy conversion efficiency of conventional PV modules. A solar position algorithm was also developed and implemented in a solar tracking system for predicting the sun position precisely [13, 14]. In addition, Akhlaghi et al. [15] proposed a new algorithm aiming to enhance the power generation of the tested solar panel by finding determined optimal periods for a given movements' number. The authors reported that adopting a solar tracking system for residential usage could be cost-inefficient. Therefore, the research was carried out to determine a specified number of an optimum tilting angle and intervals based on the forecasting model. The authors concluded that using an optimized and specified number of the tilting angles and solar tracking moves could produce a greater conversion efficiency compared to a fixed angle solar panel and instantaneous solar tracker systems. Tharamuttam et al. [16] designed and developed an automatic microcontroller-based solar tracker with a hybrid algorithm to optimize the harnessing of solar energy. The study was aiming to locate the position of the sun precisely and evaluated the performance of the proposed solar tracker system. Besides, a webpage is also developed in order to facilitate the timely monitoring of the adopted PV system performance. Moreover, a model was proposed for a two-axis solar tracking system by Aziz S. et al. [17]. The proposed system was able to communicate with the smart grid, and could also control the flow of generated power between the grid load and energy storage systems according to the electrical power demand and generation. As a result, a better energy conversion efficiency and less energy wastage were attained when the proposed system was applied.

As previously mentioned in the reviewed literatures, there are several factors that can affect the efficiency of the conventional PV system such as the sun light intensity, area of PV module, type of semiconductor, tracking mechanism, dust and dirt. To fully harness the solar energy, a better technology needs to be introduced. A conventional static solar panel is insufficient to harness the solar energy, and its conversion efficiency infrequently exceeds 19\%. This study implements a new tracking mechanism to improve the efficiency of solar energy harnessing. The implemented PV system that is combined with solar tracker generates more electricity than stationary conventional PV panel due to the increase of solar exposure. To reach the aim of this study, a fully controlled system was designed and engaged into the used PV system to track the sun position precisely and avoid and possible shadowing for the used PV panels.

\section{SUN POSITION TRACKER: SYSTEM DESCRIPTION}

The proposed Solar Panel with Sun Position Tracker (SPT) model is illustrated in Figure 1. The SPT system body frame consists of stainless-steel bar and iron rods. The overall height of the proposed system is $960 \mathrm{~mm}$ whereas the system has a side view dimension of $440 \times 586 \mathrm{~mm}$. The arrangement of the solar panels is stacked on the top of each other without overshadowing the below panels. Three rods (known as solar rods) are inserted with small bearings through stainless steel (SS) bar, which the rods are connected to solar panels and solar concentrator. Next, three servo motors are mounted on the SS at one end of each solar rod. These servo motors are connected by servo horns for a better grip. The motors help to rotate the solar panel and concentrator in horizontal axis according to specific time interval.

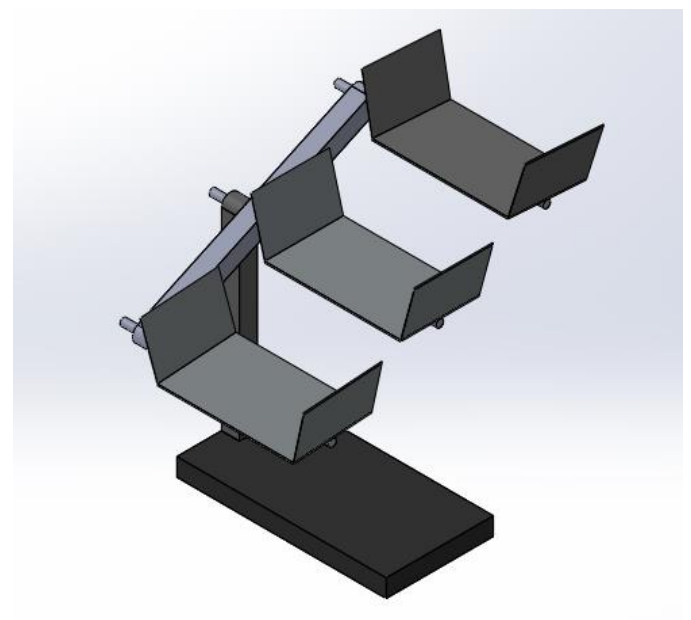

Figure 1. SolidWorks 3D drawing for the PV system with SPT model.

In the proposed system, a single SS bar is used to support all three solar panels with solar concentrators. This SS bar then is pinned to a pole of a vertical stand with a stainless steel base in accordance that SS bar is able to rotate around a 
pivot's which serves to ensure the system's stability. The rotational movement of the SS bar along a certain arc path is attained with the aid of the actuator which is placed on the pole. The actuator linear movement allows the SS bar to face either East or West according to the sun position. SS bar can rotate either East or West, hence increasing the exposure of solar panels to the solar irradiance. The most essential part of this design is the type of used solar panel. Three types of solar panels are considered in this work comprising monocrystalline silicon (Mono - Si), polycrystalline silicon ( $\mathrm{P}-\mathrm{Si}$ ) and Thin Film Solar Cells (TSFC) [9, 10]. According to the open-source literature, the polycrystalline silicon (P - Si) solar cells is selected to be the most suitable and cost-effective solar panel type for this work. Three polycrystalline silicone $(\mathrm{P}-\mathrm{Si})$ solar cells are adopted into the system with dimension $380 \times 220 \times 5 \mathrm{~mm}$ and are assembled with three MG995 servo motors with metal horns for extending the strength. The solar panels are then equipped with the solar concentrators which are flat mirrors as shown in Figure 2. These mirrors are mounted on the ends of each solar panel with a 45-degree angle slanted from the horizontal axis [11]. This is to ensure that more sun rays are concentrated on the solar panels in order to increase the penetrated solar irradiance.

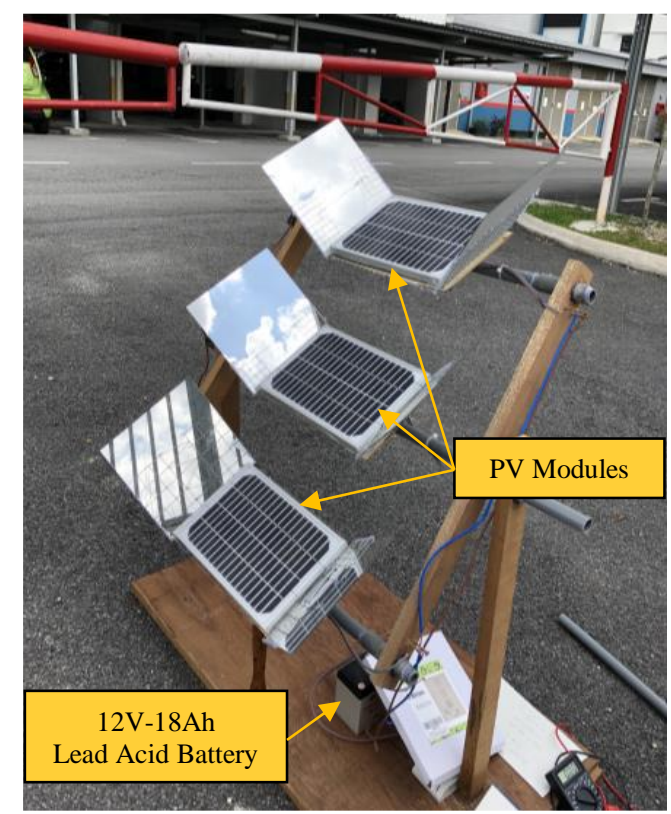

Figure 2. Prototype photo of solar panels with SPT system.

The connection of rods on the SS bar is made by drilling holes in the used SS bar where bearings are also installed. The bearings are used to ease the movement of the rods using Servo motors to rotate the PV modules according to the position of the sun. Arduino microcontroller type Mega is adopted, which has all the needed features, and it is also used as a controller for the servo motor. With Arduino Mega 2560 microcontroller, the servos are controlled to rotate the solar panels accordingly. To keep tracking of the actual date and time, a Real-Time Clock (DS3231 RTC) which is relatively cheap, and extremely accurate inter-integrated circuit real-time clock (I2C RTC) with an integrated crystal and temperature compensated crystal oscillator (TCXO) [6]. The device incorporates an independent electrical DC power source to retain accurate timekeeping even when the main supplied electrical power to the device is interrupted. The device incorporates an independent electrical DC power source to retain accurate timekeeping even when the main supplied electrical power to the device is interrupted. To determine the PV modules movement direction according to the proposed system design of this study, 12 volts DC power actuators are installed in conjunction with 2-relay modules to toggle the positive and negative power to the adopted actuators.

\section{AUTOMATED TRACKER}

The block diagram of the control circuit configuration for the SPT system is shown in Figure 3. The control circuit microcontroller acquires the data of the real-time and date from the real-time digital-clock. Then, the required time interval for the designed round movement of the used PV modules is calculated. The adopted microcontroller for this work can precisely determine the required timing for the angular and horizontal movements of the installed PV modules stack along the desired circular arc. Practically, these movements were implemented by using the aforementioned servo motors and an actuator. The adopted microcontroller is used to send the control signals to the servo motors aiming to rotate the PV panels at the precise time intervals. When the sun reaches its maximum angular position, the microcontroller will send a signal to set the middle PV module in a vertically up position to avoid any possible shading over the bottom PV module. Figure 4 shows the circuit configuration of the components based on the block diagram [18]. 


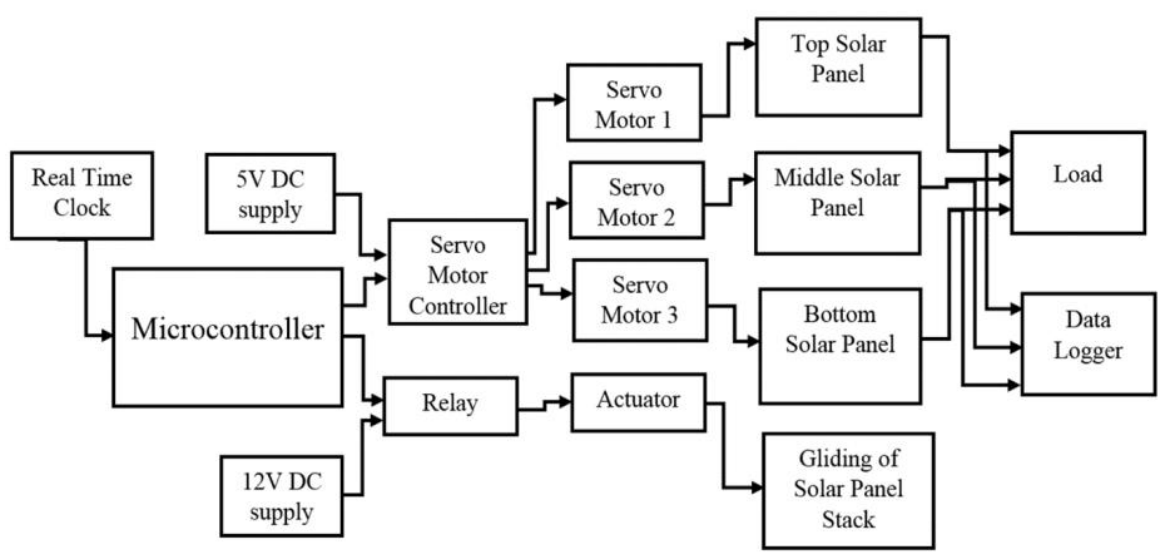

Figure 3. Block diagram of the control circuit configuration of SPT model.

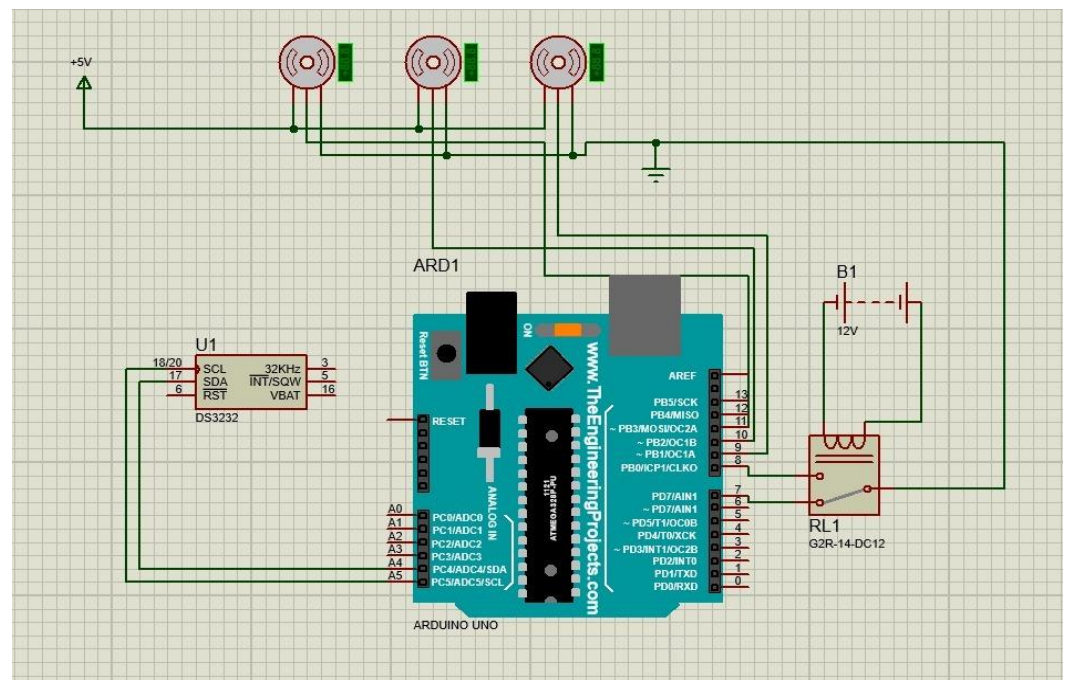

Figure 4. Circuit configuration of the SPT model.

The middle PV panel is kept static in a vertically up position until the sun moves past the zenith towards the West by the same angle. Just before noon, which is before the top and bottom PV panels start making the next rotation, the microcontroller will send the signal to activate the actuator through the relay. The actuator will swing the SS bar to the new position along East-West direction to realign the PV modules with the sun's position in the West direction in the afternoon. Therefore, the PV modules can receive much greater exposure to solar irradiance during the daytime. At sunset, the PV panels will be reset to the original positions for new testing for the next day.

\section{OPERATION}

When the day began in the morning, all the boards' components were set to face the East, and then the panels were moved from their position toward the left-hand side. The panels movement started from the base to the top according to the time set up in the control unit. The board width was considered, so every part of the boards can receive full sun exposure during the daytime, as shown in Figure 2 and Figure 5. The panels will remain at this position for a certain period before they move to the next position. In the afternoon, the center board will rotate vertically for a certain time as the sun is directly facing the SPT system. This is to avoid any possible board shading where the top and bottom boards should be fully exposed to the sunlight during the daytime hours, as shown in Figure 2 and Figure 5. The testing time for this process was achieved by the sun position for which the bottom PV panel was shadowed over by the upper PV panels. The dimensions of the inter-panel section need to be adequate enough to allow the rotation of the solar panel tracker without intersecting the course of the other panels. Accordingly, the inter-panel separation distance was set to be 1.5 times larger than the PV module width. This could enable the middle PV panel to harness a significant amount (68\%) of solar energy with a feasible PV system size [12, 19]. For a larger altitude, the magnitude of the time that the middle PV module stays in a vertical position was less energy costing compared with the generated energy due to avoiding the shadowing. In order to maximize the annual energy accumulation, the adopted PV modules were tilted at a latitude angle facing the 
South of Kajang, Malaysia. The orientation of the proposed systems' PV modules was tilted along the North-South direction.
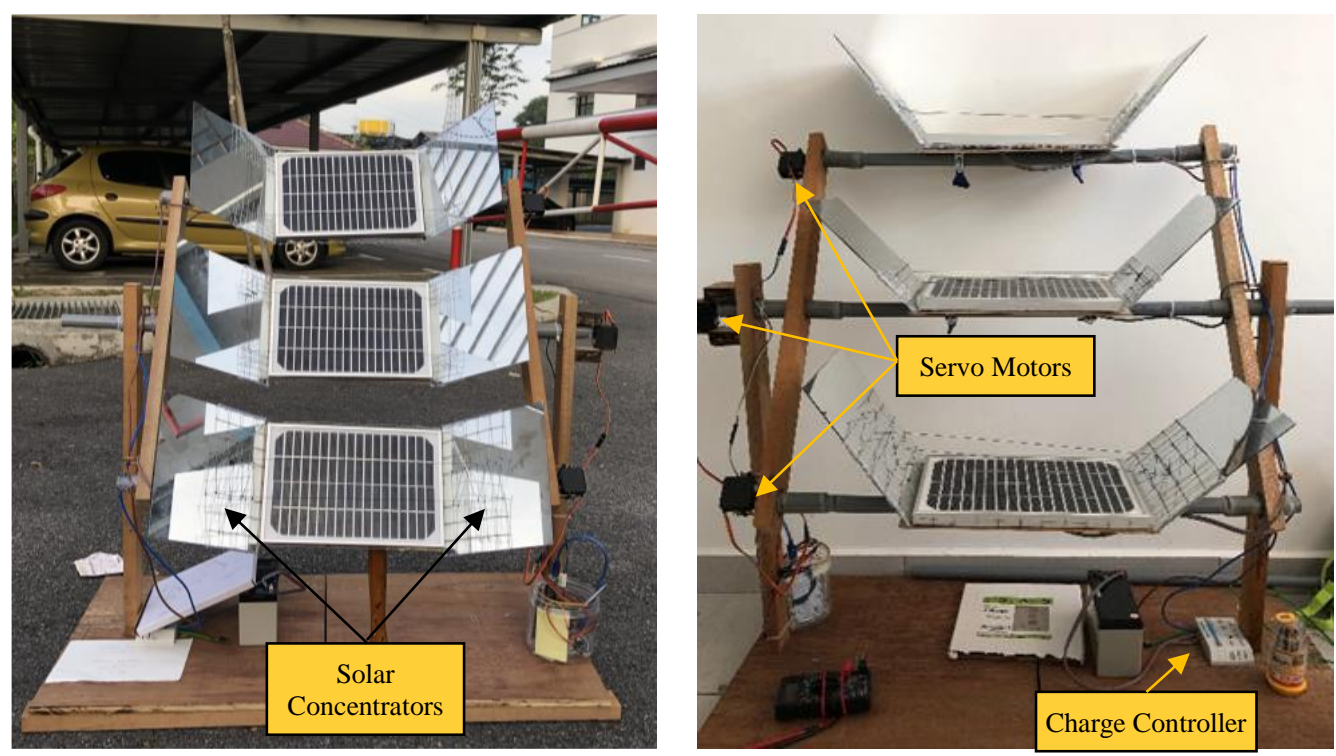

Figure 5. Prototype photo of PV modules with SPT system during the experiments.

The experimental setup was conducted in Kajang which is coordinated at $2.9787^{\circ} \mathrm{N}, 101.7348^{\circ}$ E. An open parking spot was chosen, and it is not covered with any roof or shadowed by trees. The SPT system was placed to face 108 degrees East based on the location of sun on the first day of experiment. Experiment was conducted for three consecutive days, from 14th to 16th January 2019 when the weather was changing from sunny to cloudy and vis versa during the daytime. The experiment took 10 hours starting from 8:00 AM till 06:00 PM as Malaysia receives about 10 hours of sunlight per day. To compare the results of the proposed PV system, a conventional PV panel with the same specifications as those in the multilevel system was installed at a constant position and tested together with the SPT system. The angle of the conventional panel was fixed at 15 degrees facing South which is enabling the panel facing suns path in Kajang aiming to compare the energy harnessing ability of the conventional system with the SPT system [20].

Initially, at 08:00 AM in the morning, the solar panels were facing the East by 60 degrees where the sun rises. This angle was selected as the maximum power point to harness sunlight. Since there was no data logger used in this experiment, the data was recorded manually for every 30 minutes time interval for both SPT model and static solar panel. Next, after two hours the Arduino checked the time signal from RTC and then commanded the servo motors to start rotating 30 degrees upwards. At this moment, the solar panels were almost parallel to horizontal axis.

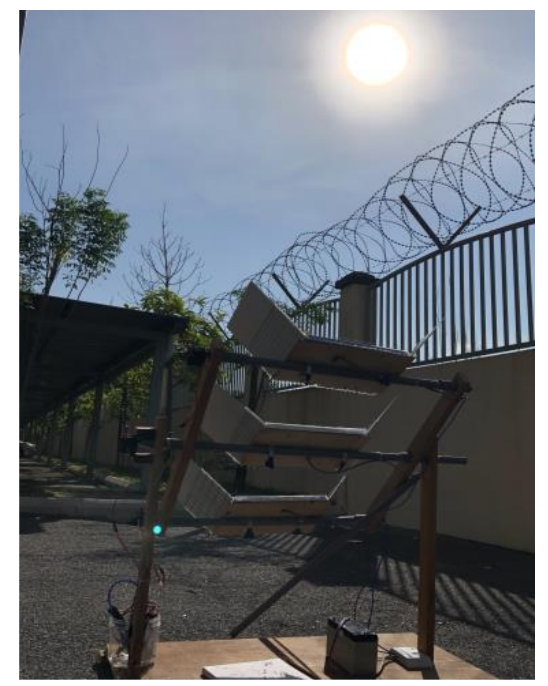

Figure 6. Prototype photo of PV modules with SPT system undergoing experiment at 08:00AM.

At 12:00 PM, a signal was sent by the Arduino to the top and bottom servo motors to rotate another $30^{\circ}$. This positioned the solar panels at the horizontal axis. This is the third position of the system. At this point, the middle Servo motor was commanded to rotate the middle solar panel into the vertical axis. This is to avoid any possible overshadowing 
to the lower solar panel as it could reduce the overall SPT system efficiency. At 02:00 PM, SS bar was flipped to the West manually while the solar panels were rotated to $30^{\circ}$ facing the West. At the end of the day at 06:00 PM, the system reset itself to the initial position and the processes were repeated day in and day out. The assembled average of three days data was considered in the results calculations and analysis. A comprehensive result analysis for the SPT system was performed and compared with the conventional tested system. A lead-acid battery with a capacity of 7.2 Ah and 18 Volt was used as a load for the system. To prevent the reverse electrical current flow from the system battery to the used PV panels in case of deficiency of the sunlight, Pulse Width Modulation (PWM) Solar Charge Controller with a capacity of 5 Volt and 1.2 Amps were installed in series connection with each panel.

\section{RESULTS AND DISCUSSIONS}

The experiments were carried out for three consecutive days which were 14,15 , and $16^{\text {th }}$ of January 2019 . The captured data of the voltage, current, and power output were analyzed and discussed. As shown in Figure 7, a comparison among the three solar panels with the solar tracker system and the static solar panels system was conducted and presented in terms of output power. The average output power was also calculated and presented in Figure 7. It can be seen that the output power generally increases for all the cases from 8.0AM reaching its maximum values at around the midday time, and then the produced power generally decreases reaching its minimum values at round 6.0PM. This could be mainly attributed to the increased solar irradiance with the rise of the sun from the early morning to the midday time. However, when the sun moves from the midday time toward the sunset time, the solar irradiance countinuously decreases reaching the lowest level at around 6.0PM. Consequently, the produced electrical power reduces correspondingly. In general, the PV system performance is significantly improved when the SPT system was implemented. As shown in Figure 7, the maximum improvement occurs at 9:00AM by about 56\%, meanwhile, the overall increment is about $28.5 \%$ for all the working hours over three days' testing time. This could be attributed to two main reasons. Firstly, implementing the SPT system to the static PV system could certainly increase the solar irradiance penetration to the PV panels. This could increase the captured energy from the sun and consequently increasing the conversion efficiency and the output power. Secondly, the used solar concentrators could certainly increase the delivered solar energy to the PV panels during the operating hours. However, it worth to be mentioned that increasing the solar penetration and concentration could potentially rise the surface temperature of the PV panels which could negatively influence the system performance by reducing the voltage of the used PV panel [21]. In fact, when the ambient temperature (operating temperature) rises above the standard operating temperature $\left(\sim 25^{\circ} \mathrm{C}\right)$, the PV system performance will gradually deteriorate with the temperature increase [22]. Moreover, it can be noticed that the gained output power fluctuates with the day time, which could be mainly due to the unpredictable weather conditions.

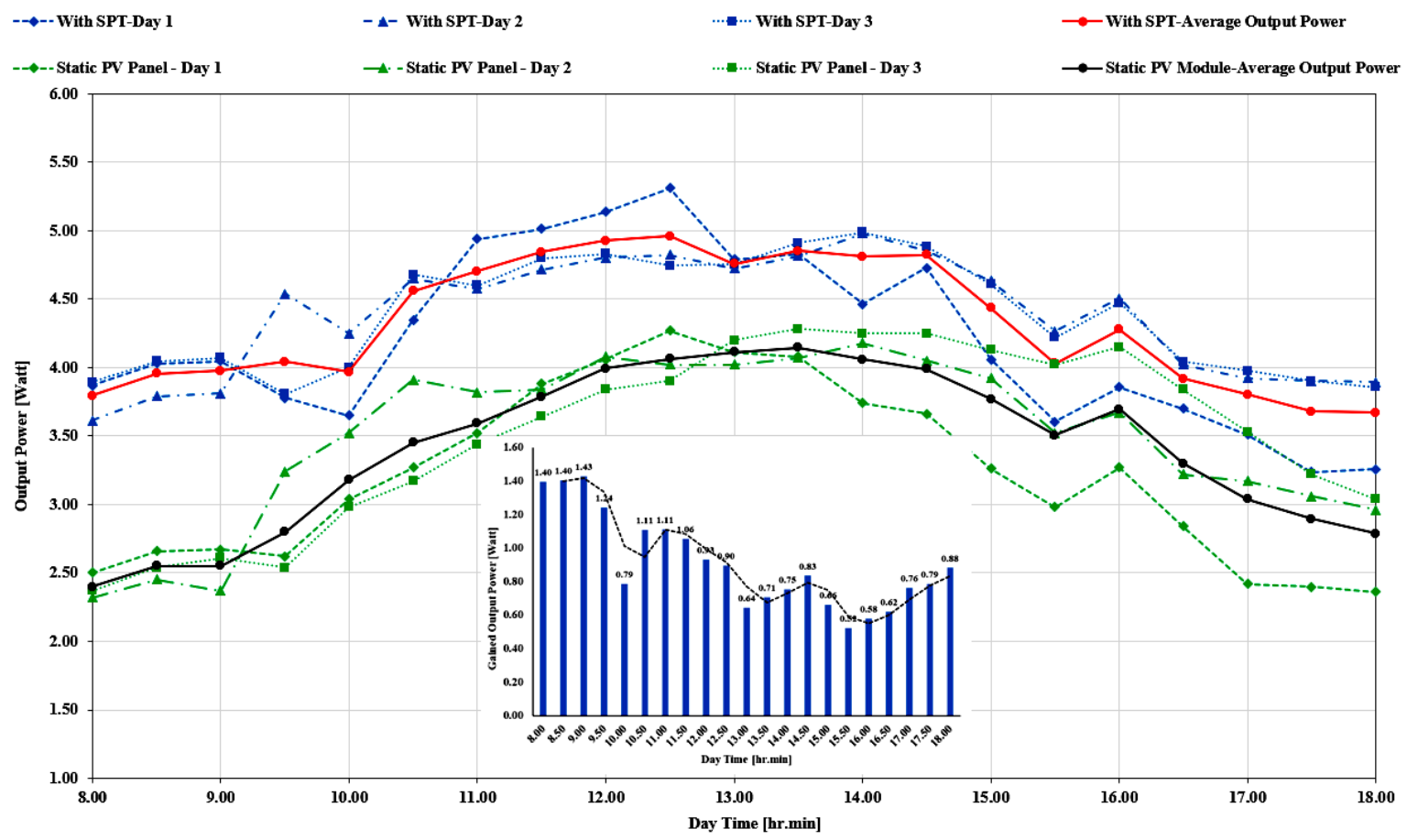

Figure 7. Real-time daily output power versus daytime for PV modules with and without the SPT system.

Figures 8, 9, and 10 illustrate the real-time captured data which represent the ensembled average results of the three days collected data of the static and rotating (accompanied with the SPT system) PV modules. As shown in Figures 8, 9, and 10, the results of the static PV module and the rotating bottom and middle PV modules in terms of output power, 
electrical current, and voltage respectively. Regarding the results of the top PV module, it was noticed that the performance of both PV modules (top and bottom) was highly similar, as shown in Figure 7. This could be due to the position and rotational movements of both panels were set the same. Therefore, the performance results of the top panel were not included in these three figures and discussions. According to Figures 8, 9, and 10, the outputs of the middle PV module is zero from 12:00 to 2:00 PM because the panel was tilted vertically upright to avoid overshadowing the bottom panel. This is due to the position of the sun during the mentioned time interval is 90 degrees above the horizon which is perpendicular to the bottom PV module.

It can be noticed that from Figure 8, the output power increases from the sunrise time to around midday reaching its peak value at around 01:00 PM. This was mainly attributed to the increased value of the solar irradiance when the sunrises from the horizon toward the vertical axis. Theoretically, with the time from around 1:00 PM until the sunset occurs at around 6:00 PM, the solar irradiance is reduced reaching its minimum value at the sunset time [23]. This can be clearly observed in the produced power of all the used PV modules. Figures 8, 9 and, 10 show that the PV module with the SPT system performs better compared with the static PV module. This superior performance is comprising the generated output power, current, and voltage. The output power increases by $19.53 \%$ at the bottom rotating PV panel compared with that of the static PV module. Moreover, a similar percentage increment by $18.66 \%$ is achieved when the rotating PV module is middle positioned. This is mainly attributed to the increased solar irradiance penetration on the rotating PV modules during day sun hours. On the other side, the conventional (static) PV module received a smaller amount of solar irradiance during the testing time with the absence of the SPT system and solar concentrators. Therefore, the PV modules with an SPT system proves its superior performance and more promising outcomes compared with the conventional PV module system.

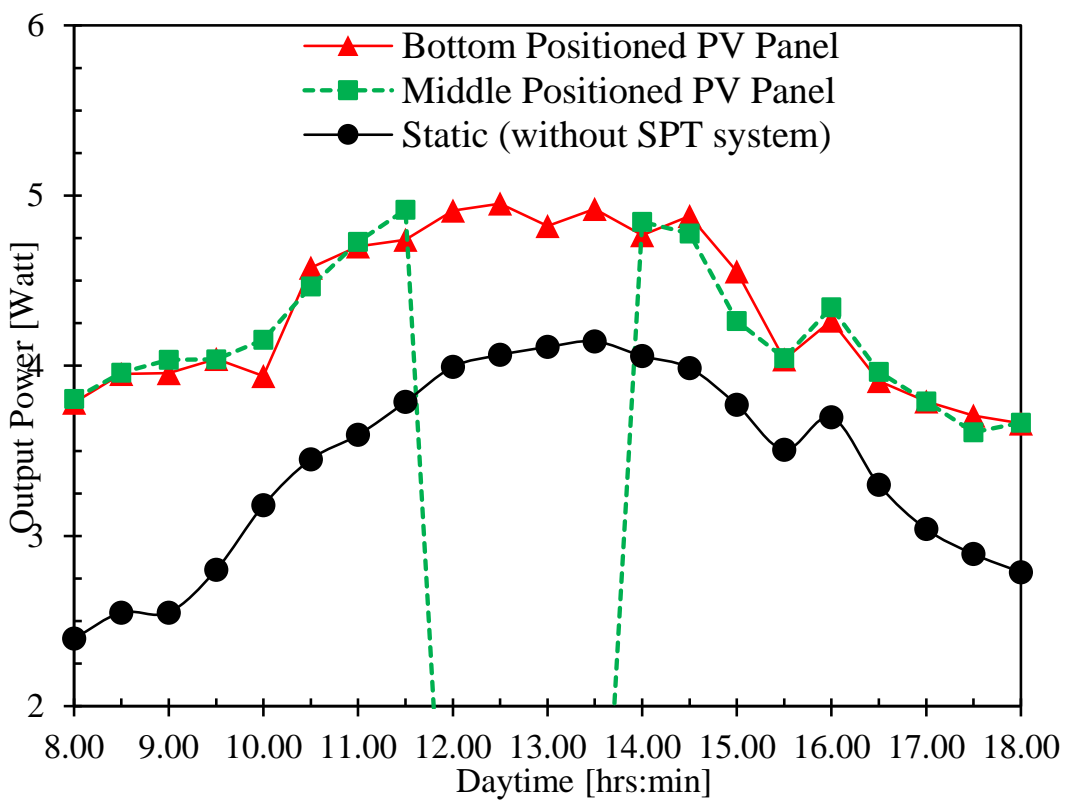

Figure 8. Variation of output power with the daytime from 8:00 AM to 6:00 PM.

Figure 9 shows that the output current gradually increases from the beginning of the experiment 8:00 AM until around 12:00 PM. From the midday time, the output current continues similar and stable at its maximum values until around 2:00 PM. After that, the current begins to decline from around 2:00 PM until the end of the experiment at 6:00 PM. This scenario could be deduced due to the location and the solar irradiance exposure on the used PV panels. It may be mentioned that the middle PV panel was not produced any current as it is tilted vertically to avoid any possible overshadowing occurrence on the bottom panel. Therefore, it can be observed that the static PV module average current output is much lower than that of the solar panels with the SPT system. The superior performance of the PV panels with the SPT system could be attributed primarily to the increased insolation penetration caused by the tracking system and the used solar concentrators. The effect of the operating temperature could be also perceived when the testing time was morning and the PV modules were relatively cold. Figure 9 shows that when the testing time is between 8.0-10.0 AM, the proposed PV system performs much better than the static PV module in terms of the output current. However, on the afternoon testing time when the PV modules were relatively warm, the proposed PV system performs differently, and the output current for both systems is almost the same.

The PV modules with the SPT system achieve their average peak voltage value of 18.0 volts when the exposure of the sun is a peak between 12:00 PM and 2:00 PM based on the obtained data. After 2:00 PM the output voltage starts decreasing with daytime reaching its minimum value at the finishing time of the conducted experiments at around 6.00PM. The static PV module also demonstrates a similar trend to that of rotating PV modules during the daytime. It can be seen that from Figure 10, the average output voltage continues increasing with the time reaching its peak value at around noontime, and it is continuing with similar output until around 2.00 PM. After that, the output voltage continues 
to reduce reaching its minimum value at the sunset time, which is similar to the produced current and power. Compared with the PV modules with the SPT system, the conventional PV module shows less promising performance in terms of output power, voltage, and current. Nevertheless, when the testing time is between 1.0 PM to 3.0 PM, the static PV module performs similar or even better than the PV modules that are equipped with the tracking system and solar concentrators. This performance can be mainly imputed to the increase in the surface temperature of the proposed PV system, which could strongly reduce the output voltage. On the other side, as shown in Figure 9, the output current of the proposed PV system is noticeably increased at the same testing time (from 1.0 PM - 3.0 PM). Fundamentally, the voltage of the PV modules decreases with the increase of the operating temperature meanwhile the output current increases [24, $25]$.

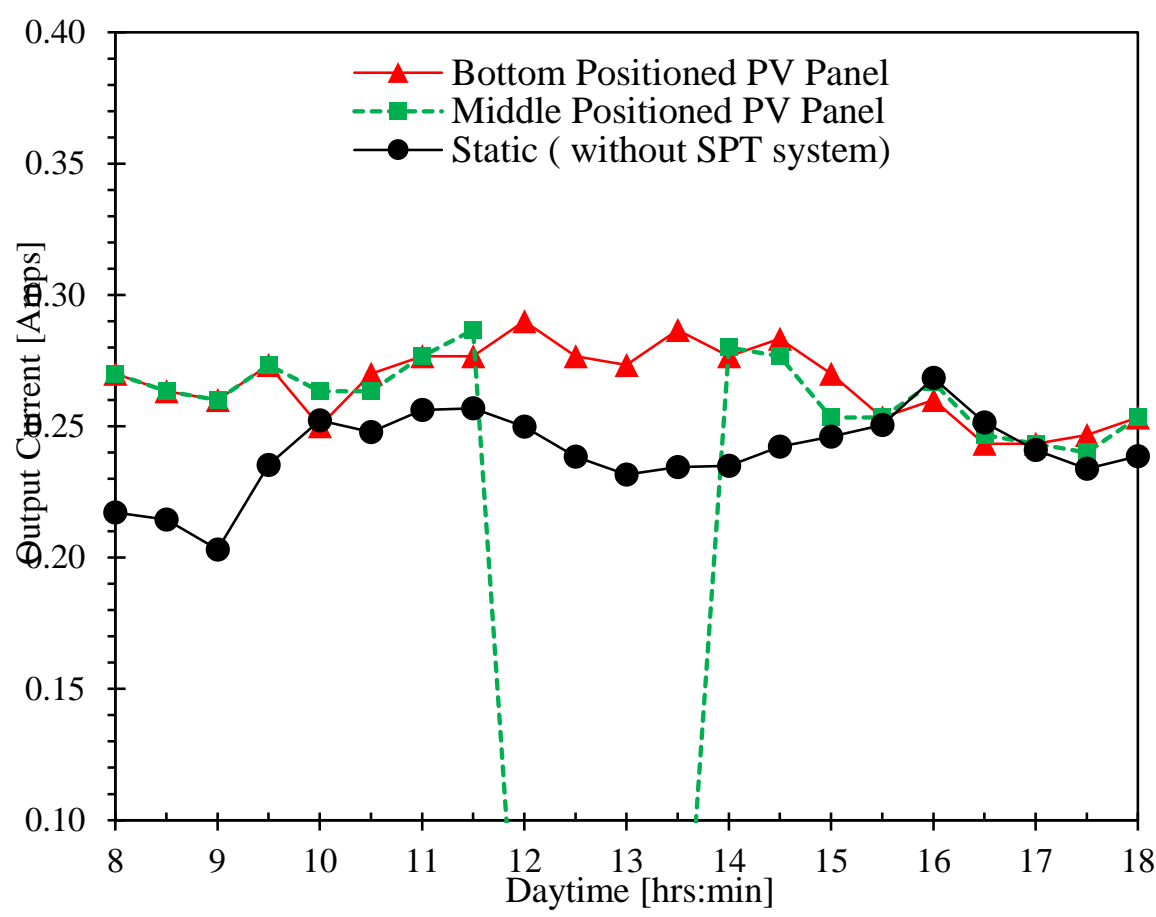

Figure 9. Variation of electrical current with the daytime from 8:00 AM to 6:00 PM.

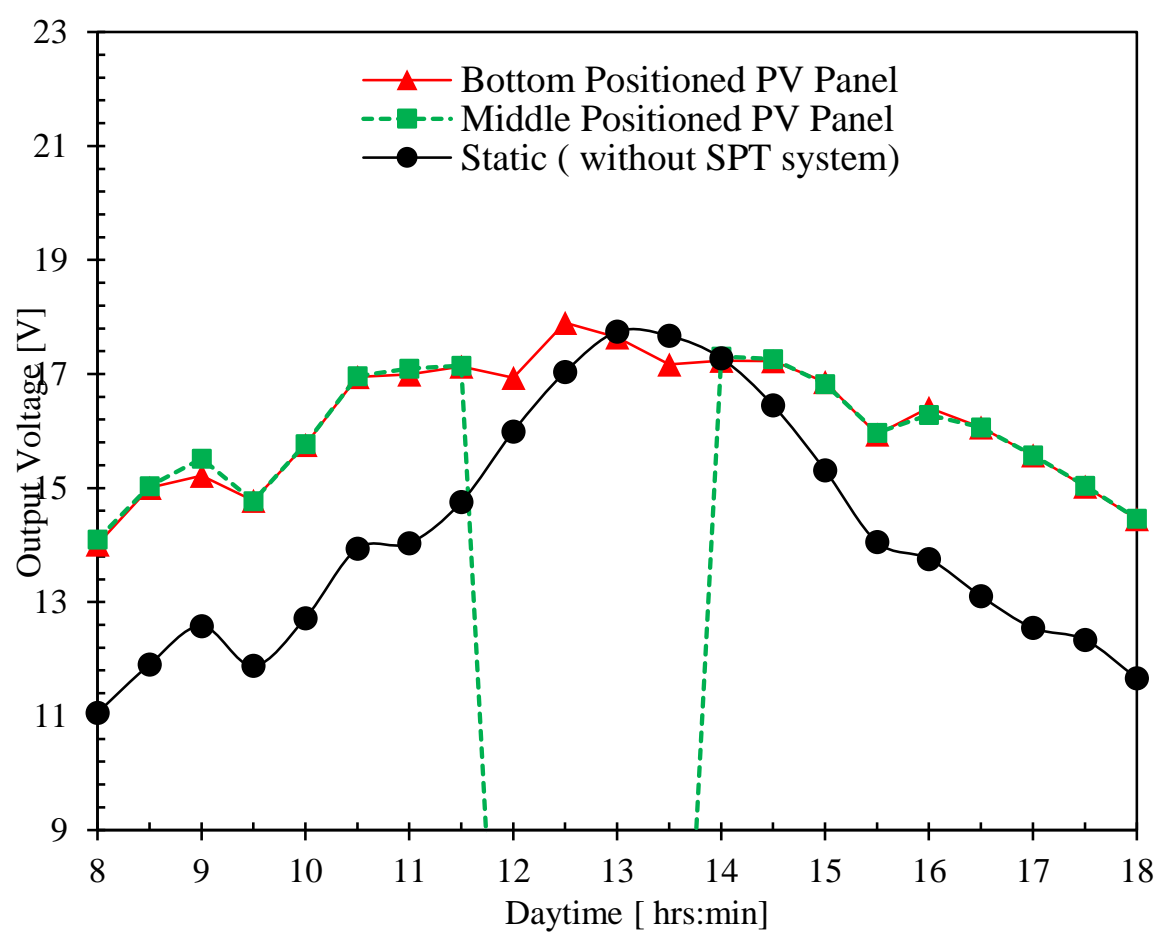

Figure 10. Variation of output voltage with the daytime from 8:00 AM to 6:00 PM. 
The resemblance of the outputs from Figures 8, 9, and 10 as the values demonstrated from the figures are strongly connected. This is because the power obtained the PV modules with the SPT system aid performs much better than the conventional (static) PV panel in terms of output power, current, and voltage. Therefore, the output voltage is directly proportional to the output current and power. However, comparing the performance of both systems with the designed (nameplate) performance of the used PV panels, it can be noticed that the attained performance from this experimental study is still below the nameplate of the used PV modules. The drop in the outputs of the proposed system and static modules could be affected by the unpredictable weather such as the presence of the cloud which could block the exposure of solar irradiance. As a result, the PV modules with the SPT system were not able to harness the maximum penetrated solar irradiance. Furthermore, the pressure from abnormal wind speed (high turbulence) could disrupt the strength of the used motor that withstands the panel at the specified angle (position).

The cumulative energies were calculated, and the results were compared to evaluate the final performance of both PV systems (with and without the SPT system). This was conducted by assuming there are three static solar panels to be compared with three rotating solar panels in the SPT system. As shown in Figure 11, the results of the calculated cumulative energy of the SPT system is clearly greater than that of the static panels even though the middle solar panel is idled for two hours. This superior performance of the SPT system is increased with daytime reaching its greatest value at 6:00 PM, as shown in Figure 11. The maximum calculated cumulative energy output for the SPT system is approximately $280 \mathrm{Wh}$ while the conventional system is $230 \mathrm{Wh}$. This means that the performance of the PV system with the SPT system and solar concentrators is clearly performing better, by about $21.24 \%$, than the conventional system. In addition to the aim of the SPT system, this performance could be mainly attributed to the used solar concentrator which could increase the solar irradiance penetration on the used PV modules of the proposed system for this study.

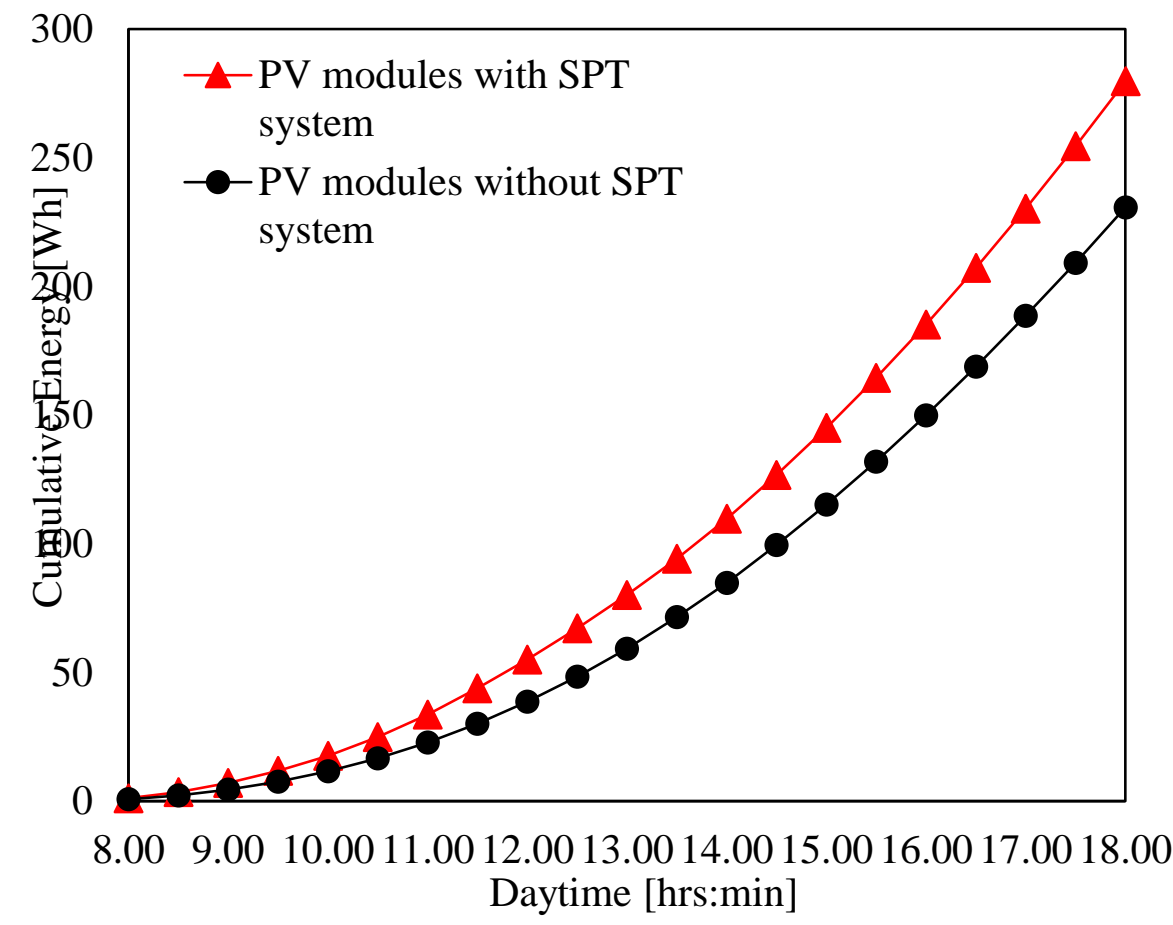

Figure 11. Cumulative energy vs daytime for PV modules with and without SPT system.

\section{CONCLUSIONS}

Multilevel solar panels system with a sun position tracker and solar concentrators was designed, fabricated and experimentally tested for three consecutive days. The proposed prototype of this work has achieved promising outputs. This was because the PV system and SPT system harvested more solar energy than that of the conventional PV system. The increase in the harnessed energy could be mainly due to the use of the SPT system and solar concentrator technology implemented.

The work achieved its objectives which firstly was studying the different working mechanisms of SPT models available in current technology. Many types of technologies were also used to improve the solar panel efficiency such as the different axis of the tracker, type of sensor used, and solar concentrator implemented. Secondly, the design and development of a better SPT system than the existing ones. It can be concluded that the betterment of this prototype is due to the installation of the solar concentrator to the PV modules equipped with the SPT system. After that, the validation of the proposed SPT model to the current conventional PV system was conducted. Experiments were carried out for three consecutive days and the average values were taken to be calculated and analysed. As forecasted, the sun position tracker with a solar concentrator yielded more solar energy than the static solar panel. The proposed system was capable to 
harness $21.24 \%$ more solar energy compared with the conventional PV system. This percentage represented about 50Wh increment in the energy generated per day when the SPT system and solar concentrators were used for the conventional PV system. Moreover, the overall increment of the average output power was about $28.5 \%$ which was calculated based on all the testing hours over three consecutive days. Besides, it was found that the performance of the proposed system could be also affected by the surface temperature of the PV modules.

\section{ACKNOWLEDGMENTS}

The authors would like to express gratitude to Power Generation Unit, Institute of Power Engineering (IPE), Universiti Tenaga Nasional (UNITEN), and Tenaga Nasional Berhad (TNB) for providing research grant to carry out this research.

\section{REFERENCES}

[1] L. Richardson. "What is the history of solar energy and when were solar panels invented?" energysage. https://news.energysage.com/the-history-and-invention-of-solar-panel-technology/ (accessed 4th May 2018).

[2] M. M. Shivamallaiah et al., "Experimental study of the influence of glass cover cooling using evaporative cooling process on the thermal performance of single basin solar still," Journal of Mechanical Engineering and Sciences, vol.14, pp. 6334-6343.

[3] A. M. Muzathik, W. M. N. B. W. Nik, K. B. Samo, and M. Ibrahim, "Reference solar radiation year and some climatology aspects of East Coast of West Malaysia," American Journal of Engineering and Applied Sciences, vol.3, pp. 293 - 299 , doi:http://ir.lib.seu.ac.lk/handle/123456789/2513.

[4] M. Abid, B. Yousef, M. Assad, A. Hepbasli, and K. Saeed, "An experimental study of solar thermal system with storage for domestic applications," Journal of Mechanical Engineering and Sciences, vol.12, pp. 4098-4116.

[5] M. Manjunath, R. Venkatesh, and N. Madhwesh, "Thermal performance enhancement of flat plate solar air heater using transverse U-shaped turbulator-A numerical study," Journal of Mechanical Engineering and Sciences, vol.13, pp. 5562-5587.

[6] N. A. Satwika, R. Hantoro, E. Septyaningrum, and A. Mahmashani, "Analysis of wind energy potential and wind energy development to evaluate performance of wind turbine installation in Bali, Indonesia," Journal of Mechanical Engineering and Sciences, vol.13, pp. 4461-4476.

[7] M. E. H. Chowdhury, A. Khandakar, B. Hossain, and R. Abouhasera, "A Low-Cost Closed-Loop Solar Tracking System Based on the Sun Position Algorithm," Journal of Sensors, vol.2019, pp. 3681031, doi:https://doi.org/10.1155/2019/3681031.

[8] T. Debnath, S. N. Imtiaz, and M. Rahman, "Implementation of an RTC based multilevel solar panel system," in IEEE Region 10 Humanitarian Technology Conference (R10-HTC), Dhaka, Bangladesh, Dec. 2017 2017, pp. 610 - 614, doi: 10.1109/R10HTC.2017.8289034.

[9] M. S. Kabir, R. R. Chowdhury, M. N. Amin, D. M. Abdullah, W. M. Abdullah, and M. A. Arafat, "Solar power enhancement using concentration method, tracking system and fuzzy based control system," in 15th International Conference on Computer and Information Technology (ICCIT), Dec. 2012 2012, pp. 464 - 468, doi: 10.1109/ICCITechn.2012.6509737.

[10] V. Sharma and V. K. Tayal, "Hardware implementation of sun tracking solar panel using 8051 micro-controller," in 6th International Conference on Reliability, Infocom Technologies and Optimization (Trends and Future Directions) (ICRITO), September 20172017 pp. 483 - 486, doi: 10.1109/ICRITO.2017.8342475.

[11] W. Liu, "Sun Tracker: Design, Build and Test," in IEEE 81st Vehicular Technology Conference (VTC Spring), Glasgow, United Kingdom, May 2015 2015, pp. 1-5, doi: 10.1109/VTCSpring.2015.7146115.

[12] S. Meikap and S. Dey, "Development of a low-cost optimum power tracking prototype for solar energy," in IEEE Annual India Conference (INDICON), Dec. 2016 2016, pp. 1 - 5, doi: 10.1109/INDICON.2016.7839013.

[13] Y. Rizal and S. H. Wibowo, "Application of solar position algorithm for sun-tracking system," Energy Procedia, vol.32, pp. 160 - 165, doi:https://doi.org/10.1016/j.egypro.2013.05.021.

[14] K. B. de Melo, M. Kitayama, L. R. Tavares, H. A. de Freitas, and M. G. Villalva, "Accuracy Analysis of Sun Position Calculation Algorithms: Ineichen and SPA," in 2019 IEEE PES Innovative Smart Grid Technologies Conference-Latin America (ISGT Latin America), 2019: IEEE, pp. 1-5.

[15] S. Akhlaghi, H. Sangrody, M. Sarailoo, and M. Rezaeiahari, "Efficient operation of residential solar panels with determination of the optimal tilt angle and optimal intervals based on forecasting model," IET Renewable Power Generation, vol.11, pp. 1261 - 1267, doi:10.1049/iet-rpg.2016.1033.

[16] J. K. Tharamuttam and A. K. Ng, "Design and Development of an Automatic Solar Tracker," Energy Procedia, vol.143, pp. 629 - 634, doi:https://doi.org/10.1016/j.egypro.2017.12.738.

[17] S. Aziz and S. Hassan, "On improving the efficiency of a solar panel tracking system," Procedia Manufacturing, vol.7, pp. 218 - 224, doi:https://doi.org/10.1016/j.promfg.2016.12.053.

[18] A. H. Zenan, S. Ahmed, M. K. Rhaman, and M. Rahman, "A new multilevel solar panel system for urban areas," in 2013 IEEE 39th Photovoltaic Specialists Conference (PVSC), June 2013 2013, pp. 1526 - 1530, doi: 10.1109/PVSC.2013.6744435.

[19] M. Boxwell, Solar Electricity HandBook, 17th ed. ed. (17 ${ }^{\text {th }}$ Edition). Birmingham: Olton Boulevard East, UK: GreenStream Publishing Ltd., 2017. 
[20] H. M. Moniruzzaman, M. Patwary, and M. Rahman, "A three level solar panel system with an automated solar tracker," in $3 r d$ International Conference on the Developments in Renewable Energy Technology (ICDRET), May 2014 2014, pp. 1 - 7, doi: 10.1109/ICDRET.2014.6861671.

[21] E. Pinheiro, F. Bandeiras, M. Gomes, P. Coelho, and J. Fernandes, "Performance analysis of wind generators and PV systems in industrial small-scale applications," Renewable and Sustainable Energy Reviews, vol.110, pp. 392-401, doi:https://doi.org/10.1016/j.rser.2019.04.074.

[22] W. Yuan et al., "Comparison study of the performance of two kinds of photovoltaic/thermal (PV/T) systems and a PV module at high ambient temperature," Energy, vol.148, pp. 1153-1161, doi:https://doi.org/10.1016/j.energy.2018.01.121.

[23] L. Al-Ghussain, H. Ahmed, and F. Haneef, "Optimization of hybrid PV-wind system: Case study Al-Tafilah cement factory, Jordan," Sustainable Energy Technologies and Assessments, vol.30, pp. 24-36, doi:https://doi.org/10.1016/j.seta.2018.08.008.

[24] A. Shukla, K. Kant, A. Sharma, and P. H. Biwole, "Cooling methodologies of photovoltaic module for enhancing electrical efficiency: A review," Solar Energy Materials and Solar Cells, vol.160, pp. 275-286, doi:http://dx.doi.org/10.1016/j.solmat.2016.10.047.

[25] V. M. Andreev, V. A. Grilikhes, and V. D. Rumiantsev, Photovoltaic conversion of concentrated sunlight. John Wiley, 1997. 